\title{
The effects of synbiotic supplementation on markers of insulin metabolism and lipid profiles in gestational diabetes: a randomised, double-blind, placebo-controlled trial
}

\author{
Shahnaz Ahmadi ${ }^{1,2}$, Mehri Jamilian ${ }^{3 *}$, Maryam Tajabadi-Ebrahimi ${ }^{4}$, Parvaneh Jafari ${ }^{5}$ and \\ Zatollah Asemi ${ }^{6}+$ \\ ${ }^{1}$ Department of Gynecology and Obstetrics, School of Medicine, Iran University of Medical Sciences, PO Box 83121625, \\ Tebran, Iran \\ ${ }^{2}$ Department of Gynecology and Obstetrics, School of Medicine, Bushehr University of Medical Sciences, Bushehr, Iran \\ ${ }^{3}$ Department of Gynecology and Obstetrics, School of Medicine, Endocrinology and Metabolism Research Center, Arak \\ University of Medical Sciences, PO Box 6618634683, Arak, Iran \\ ${ }^{4}$ Science Department, Science Faculty, Islamic Azad University, Tehran Central Branch, PO Box 83121625, Tehran, Iran \\ ${ }^{5}$ Department of Microbiology, Science Faculty, Islamic Azad University, Arak Branch, PO Box 6618634683, Arak, Iran \\ ${ }^{6}$ Research Center for Biochemistry and Nutrition in Metabolic Diseases, Kashan University of Medical Sciences, PO Box \\ 8715988141, Kashan, Iran
}

(Submitted 6 June 2016 - Final revision received 16 July 2016 - Accepted 24 August 2016 - First published online 29 September 2016)

\section{Abstract}

To the best of our knowledge, data on the effects of synbiotic supplementation on markers of insulin metabolism and lipid concentrations in patients with gestational diabetes mellitus (GDM) are scarce. The aim of the current study was to determine the effects of synbiotic supplementation on markers of insulin metabolism and lipid profiles in GDM patients. In total, seventy patients with GDM aged 18-40 years were assigned to two groups - the synbiotic group ( $n$ 35) and the placebo group ( $n$ 35) - in this randomised, double-blind, placebo-controlled trial. Patients in the synbiotic group received a daily capsule that contained three viable and freeze-dried strains: Lactobacillus acidophilus, Lactobacillus casei and Bifidobacterium bifidum $\left(2 \times 10^{9}\right.$ colony-forming units/g each) plus $800 \mathrm{mg}$ inulin for 6 weeks. Fasting blood samples were collected at the beginning and week 6 to quantify related markers. After 6 weeks of intervention, compared with the placebo, synbiotic supplementation led to a significant decrease in serum insulin levels ( -1.5 (sD 5.9$)$ $v .+4.8(\mathrm{sD} 11.5) \mu \mathrm{IU} / \mathrm{ml}, P=0.005)$, homoeostatic model assessment for insulin resistance $(-0.4(\mathrm{SD} 1 \cdot 3) v .+1 \cdot 1 \quad(\mathrm{sD} 2 \cdot 7), P=0.003)$ and homoeostatic model assessment for $\beta$ cell function $(-5.1$ (SD 24.2) $v++18.9$ (SD 45.6), $P=0.008)$ and a significant increase in quantitative insulin sensitivity check index $(+0.01$ (SD 0.01) $v .-0.007$ (sD 0.02), $P=0.02$ ). In addition, synbiotic intake significantly decreased serum TAG $(-14.8$ (sD 56.5) $v .+30 \cdot 4(\mathrm{sD} 37 \cdot 8) \mathrm{mg} / \mathrm{dl}, P<0 \cdot 001)$ and VLDL-cholesterol concentrations (-3.0 (sD 11.3) $v$. $+6 \cdot 1$ (sD 7.6) mg/dl, $P<0.001)$ compared with the placebo. Overall, the results of this study demonstrate that taking synbiotic supplements for 6 weeks among patients with GDM had beneficial effects on markers of insulin metabolism, TAG and VLDL-cholesterol concentrations.

Key words: Synbiotic supplementation: Gestational diabetes mellitus: Insulin resistance: Lipid profiles

Gestational diabetes mellitus (GDM), carbohydrate intolerance and insulin resistance during pregnancy are serious problems that are increasing worldwide, which carry significant shortterm and long-term adverse health outcomes in both mother and offspring ${ }^{(1)}$. In GDM women, the physiological changes in insulin resistance and lipid profiles are exaggerated and may indicate an underlying metabolic dysfunction that transiently manifests during pregnancy ${ }^{(2)}$. In addition, a few studies have postulated that changes in the human gut microbiome are associated with metabolic diseases including type 2 diabetes mellitus (T2DM), insulin resistance ${ }^{(3)}$ and increased inflammatory cytokines ${ }^{(4)}$.

Abbreviations: CFU, colony-forming units; FOS, fructo-oligosaccharides; FPG, fasting plasma glucose; GDM, gestational diabetes mellitus; HOMA-IR, homoeostasis model of assessment for insulin resistance.

* Corresponding authors: Z. Asemi, fax +98 315546 3377, email asemi_r@yahoo.com; M. Jamilian, email jamilian.mehri@gmail.com

$\dagger$ Present address: Department of Nutrition, Kashan University of Medical Sciences, Kashan, Iran. 
The potential role of the gut flora as well as synbiotics in decreased insulin resistance and decreased levels of cholesterol and TAG resulted in an interest in using synbiotics as preventive and therapeutic interventions. Probiotics are live bacteria and yeasts that confer health benefits to the host when consumed in sufficient quantities ${ }^{(5)}$. Furthermore, prebiotics such as inulin and fructo-oligosaccharides (FOS) that stimulate the growth and metabolism of probiotics in the gut have also been found to a exhibit favourable role against several gut-related complications ${ }^{(6)}$. The beneficial effects of synbiotics on markers of insulin metabolism and lipid concentrations in patients with $\mathrm{T}_{2} \mathrm{DM}^{(7)}$ and in subjects with the metabolic syndrome ${ }^{(8)}$ have been previously reported. We have shown that consumption of a synbiotic food containing Lactobacillus sporogenes $\left(18 \times 10^{7}\right.$ colony-forming units (CFU)) plus $0.72 \mathrm{~g}$ inulin for 9 weeks by pregnant women had beneficial effects on markers of insulin metabolism, but did not affect fasting plasma glucose (FPG) concentrations $^{(9)}$. In addition, a significant reduction in total cholesterol, LDL-cholesterol and TAG and an increase in HDLcholesterol were observed following the consumption of synbiotics containing Lactobacillus salivarius with FOS among healthy, young individuals for 6 weeks, but it did not influence insulin resistance ${ }^{(10)}$.

Intake of synbiotics may improve markers of insulin resistance and lipid profiles in GDM patients through the production of SCFA, carbon disulfide and methyl acetate ${ }^{(11)}$ and decreased expression of inflammation-related genes ${ }^{(12)}$. To our knowledge, data on the effects of synbiotic supplementation on markers of insulin metabolism and lipid concentrations in patients with GDM are scarce. The objective of this study was to evaluate the effects of synbiotic supplementation on markers of insulin metabolism and lipid profiles in these patients.

\section{Methods}

\section{Trial design}

The present study was a 6-week prospective, randomised, double-blind, placebo-controlled clinical trial.

\section{Participants}

In the present study, we included seventy women with GDM aged 18-40 years without previous diabetes, who were diagnosed with GDM by 'one-step', 2-h, 75-g oral glucose tolerance test (OGTT) at 24-28 weeks of gestation, referred to Kosar Clinic in Arak, Iran, from March 2016 to May 2016. We diagnosed GDM on the basis of the American Diabetes Association guidelines ${ }^{(13)}$. Patients who met one of the following criteria were considered as having GDM: FPG $\geq 92 \mathrm{mg} / \mathrm{dl}$, 1-h OGTT $\geq 180 \mathrm{mg} / \mathrm{dl}$ and 2 -h OGTT $\geq 153 \mathrm{mg} / \mathrm{dl}^{(13)}$. Exclusion criteria were as follows: subjects taking synbiotic or probiotic supplements, including probiotic yogurt, kefir and other fermented foods, subjects taking insulin, subjects with placenta abruption, pre-eclampsia, eclampsia, hypothyroidism and hyperthyroidism, smokers, and those with kidney or liver diseases.

\section{Ethics statements}

This trial was performed according to the principles of the Declaration of Helsinki, and the study protocol was approved by the ethics committee of AUMS (reference number IR.ARAKMU.REC.1395.22). The study protocol was carefully explained to all subjects before obtaining informed consent. The current study is registered in the Iranian website (www. irct.ir) for registration of clinical trials (http://www.irct.ir: IRCT201605085623N77).

\section{Study design}

At the onset of the study, patients were first matched one by one according to age and BMI. Next, the matched patients were randomly assigned to the intervention and placebo groups. The participants were randomly allocated into two treatment groups to take either synbiotic supplements ( $n$ 35) or placebo ( $n$ 35) for 6 weeks. At the onset of the study, patients were requested not to change their routine physical activity or usual dietary patterns throughout the study and not to consume any supplements other than the ones provided to them by the investigators, as well as not to take any medications that might affect findings during the 6-week intervention. Dietary macronutrient and micronutrient intakes were assessed using 3-d food records (comprised of 2 working days and a weekend day) at study onset, weeks 3 and 5, and at the end of the intervention. All participants completed the physical activity records at weeks 0 , 3 and 6 of the intervention. Modified Nutritionist- 4 software programme (First Databank) was used to estimate the energy and nutrient intakes. In the present study, physical activity was described as metabolic equivalents (MET) in $\mathrm{h} / \mathrm{d}$. To determine the MET for each patient, we multiplied the times (h/d) reported for each physical activity by the related MET coefficient using standard tables ${ }^{(14)}$. Questionnaires were used to measure physical activity levels.

\section{Intervention}

The synbiotic capsules contained Lactobacillus acidopbilus, Lactobacillus casei and Bifidobacterium bifidum $\left(2 \times 10^{9} \mathrm{CFU} / \mathrm{g}\right.$ each) plus $0.8 \mathrm{~g}$ inulin. Participants in the placebo group received capsules containing starch without bacteria and inulin. It is well known that it would be more appropriate if the strains used in probiotic supplements for human consumption are derived from the human intestinal tract, are well characterised, are able to outlive the rigours of the digestive tract and possibly colonise, are biologically active against the target, and are stable and amenable to commercial production and distribution ${ }^{(15)}$. Owing to the lack of evidence about the appropriate dosage of probiotics and inulin for GDM women, we used the abovementioned doses of probiotics based on a few previous studies in healthy subjects ${ }^{(16,17)}$, and the dose of inulin was based on a previous study in healthy pregnant women ${ }^{(9)}$. The appearance of the placebo was indistinguishable with regard to colour, shape, size and packaging, smell and taste from the probiotic capsule. All capsules were produced by Tak Gen Zist 
Pharmaceutical Company, which is approved by Food and Drug Administration.

\section{Treatment adherence}

Every 2 weeks, individuals were provided sufficient synbiotic and placebo capsules to last $3 \mathrm{~d}$ after their next scheduled visit and were instructed to return all unused supplements at each visit. The remaining supplements were counted and subtracted from the number provided to determine the number taken. To increase compliance, all participants received short messages on their cell phones as a reminder to take the supplements every day.

\section{Assessment of anthropometric measurements}

Weight and height of participants were determined in an overnight fasting status using a standard scale (Seca) at the onset of the study and after 6 weeks' intervention. BMI was calculated using the height and weight measurements (weight in $\mathrm{kg} /\left(\right.$ height in metres) ${ }^{2}$ ).

\section{Assessment of outcomes}

In the present study, the primary outcome measurements were markers of insulin metabolism, and the secondary outcome measurements were lipid concentrations.

\section{Biochemical assessment}

Blood samples were collected at weeks 0 and 6 after at least $12 \mathrm{~h}$ of fasting. All blood samples were centrifuged at $3000 \mathrm{~g}$ for $10 \mathrm{~min}$, and serum samples were separated into clean tubes and stored at $-80^{\circ} \mathrm{C}$ until analysis at the AUMS reference laboratory. To determine FPG, serum TAG, VLDL-cholesterol, totalcholesterol, LDL-cholesterol and HDL-cholesterol concentrations, we used enzymatic kits (Pars Azmun). All inter- and intra-assay coefficients of variances (CV) for FPG and lipid concentrations were $<5 \%$. Circulating levels of serum insulin were determined using ELISA kits (Monobind) with intra- and inter-assay CV of $3 \cdot 0$ and $4.7 \%$, respectively. The homoeostatic model assessment for insulin resistance (HOMA-IR), homoeostatic model assessment for B-cell function (HOMA-B) and the quantitative insulin sensitivity check index (QUICKI) were determined according to suggested formulae ${ }^{(18)}$.

\section{Sample size}

To calculate sample size, we used the standard formula suggested for parallel clinical trials after considering type I error $(\alpha)$ of 0.05 and type II error $(\beta)$ of 0.20 (power $=80 \%$ ). On the basis of a previous study ${ }^{(9)}$, we used 1.8 as SD and 1.3 as the difference in mean $(d)$ of HOMA-IR as key variables. Therefore, we needed thirty persons in each group. Assuming five dropouts in each group, the final sample size was determined to be thirtyfive subjects per group.

\section{Randomisation}

Randomisation was carried out using computer-generated random numbers. Randomisation and allocation were concealed from the researchers and participants until all the analyses were completed. The randomised allocation sequence, enrolling participants and allocating them to interventions were conducted by a trained staff at the gynaecology clinic.

\section{Statistical methods}

Normality variables were evaluated by the Kolmogorov-Smirnov test. All values are expressed as means and standard deviations. The data were analysed according to the intentionto-treat (ITT) principle using the Statistical Package for Social Science, version 18 (SPSS Inc.). Differences in general characteristics and daily macronutrient and micronutrient intakes between the two groups were tested by independent sample $t$ test before and after intervention. Differences within a group before and after intervention were analysed by paired sample $t$ test. To determine the effects of synbiotic intake on markers of insulin resistance and lipid concentrations, one-way, repeatedmeasures ANOVA was used to evaluate the between-group changes in variables during the study. To assess whether the magnitude of the change depended on the baseline values of biochemical parameters, maternal age and baseline BMI, we adjusted all analyses for these variables to avoid the potential bias that might have resulted. These analyses were carried out using ANCOVA. $P$ values $<0.05$ were considered to be statistically significant.

\section{Results}

At baseline, we recruited ninety patients; however, twenty subjects were excluded from the study as they did not meet inclusion criteria. As demonstrated in the study flow diagram (Fig. 1), during the intervention phase of the study, three participants were excluded from the placebo group (withdrawn because of personal reasons ( $n 3)$ ). In addition, one subject was excluded from the synbiotic group (withdrawn because of personal reasons $(n$ 1)). However, as the analysis was performed on the basis of the ITT principle, all seventy subjects with GDM were included in the final analysis. On average, the rate of compliance in our study was high, as more than $90 \%$ of the probiotic capsules were consumed throughout the study in both groups. No side-effects were reported following supplementation with synbiotics in GDM patients throughout the study.

Mean age, height, baseline weight and BMI as well as their means after 6 weeks of intervention were not significant between the synbiotic group and the placebo group (Table 1).

Comparison of dietary $3-\mathrm{d}$ intakes of study participants throughout the study revealed no significant differences in macronutrient and micronutrient intakes including energy, carbohydrates, proteins, fats, SFA, PUFA, MUFA, cholesterol, total dietary fibre, $\mathrm{Fe}, \mathrm{Mg}, \mathrm{Zn}$ and $\mathrm{Mn}$ between the two groups (Table 2). 


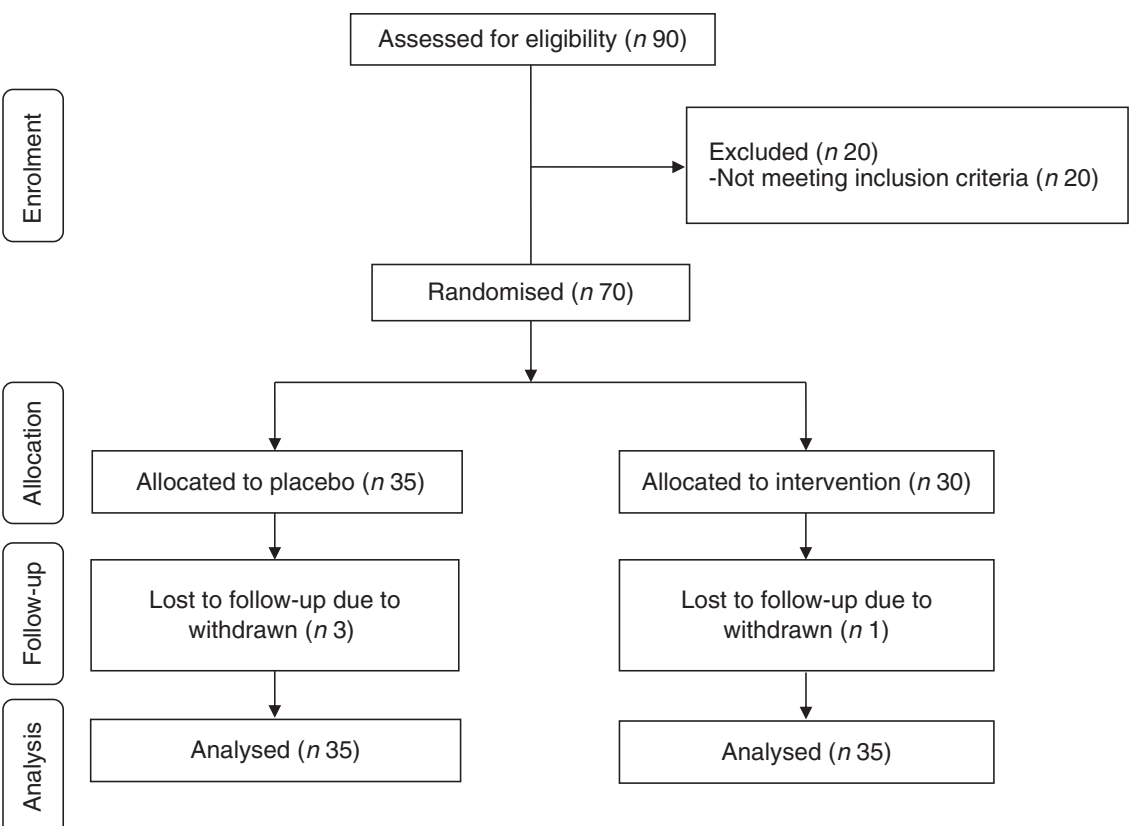

Fig. 1. Summary of patient flow.

Table 1. General characteristics of the study participants (Mean values and standard deviations)

\begin{tabular}{|c|c|c|c|c|c|}
\hline & \multicolumn{2}{|c|}{ Placebo group ( $n$ 35) } & \multicolumn{2}{|c|}{ Synbiotic group ( $n$ 35) } & \multirow[b]{2}{*}{$P^{\star}$} \\
\hline & Mean & SD & Mean & SD & \\
\hline Age (years) & 28.7 & 3.4 & 28.5 & $5 \cdot 8$ & 0.86 \\
\hline Height $(\mathrm{cm})$ & 162.6 & 4.0 & $164 \cdot 6$ & $6 \cdot 1$ & 0.09 \\
\hline Weight at study baseline $(\mathrm{kg})$ & 74.9 & $6 \cdot 6$ & $77 \cdot 7$ & $12 \cdot 6$ & 0.24 \\
\hline Weight at the end of the trial $(\mathrm{kg})$ & $77 \cdot 2$ & 6.5 & $80 \cdot 3$ & $12 \cdot 3$ & 0.19 \\
\hline Weight change $(\mathrm{kg})$ & $2 \cdot 3$ & 1.3 & $2 \cdot 6$ & 0.8 & 0.28 \\
\hline BMI at study baseline $\left(\mathrm{kg} / \mathrm{m}^{2}\right)$ & 28.4 & $2 \cdot 7$ & $28 \cdot 7$ & 4.5 & 0.73 \\
\hline $\mathrm{BMl}$ at the end of the trial $\left(\mathrm{kg} / \mathrm{m}^{2}\right)$ & 29.2 & $2 \cdot 6$ & $29 \cdot 6$ & $4 \cdot 3$ & 0.65 \\
\hline BMl change $\left(\mathrm{kg} / \mathrm{m}^{2}\right)$ & $0 . \overline{9}$ & 0.5 & 0.9 & 0.3 & 0.39 \\
\hline
\end{tabular}

* Obtained from independent $t$ test.

Table 2. Dietary intakes of study participants throughout the study (Mean values and standard deviations)

\begin{tabular}{|c|c|c|c|c|c|}
\hline & \multicolumn{2}{|c|}{ Placebo group ( $n$ 35) } & \multicolumn{2}{|c|}{ Synbiotic group (n 35) } & \multirow[b]{2}{*}{$P^{\star}$} \\
\hline & Mean & SD & Mean & SD & \\
\hline Energy (kJ/d) & 10201 & 778 & 10058 & 854 & \\
\hline Energy (kcal/d) & 2438 & 186 & 2404 & 204 & 0.45 \\
\hline Carbohydrates (g/d) & $332 \cdot 1$ & 34.0 & 328.5 & 45.4 & 0.69 \\
\hline Protein $(\mathrm{g} / \mathrm{d})$ & 88.1 & $12 \cdot 4$ & 84.6 & $16 \cdot 8$ & 0.30 \\
\hline Fat $(g / d)$ & 88.1 & $14 \cdot 3$ & $87 \cdot 2$ & $17 \cdot 2$ & 0.81 \\
\hline SFA $(g / d)$ & $25 \cdot 0$ & $5 \cdot 7$ & $25 \cdot 3$ & $6 \cdot 3$ & 0.82 \\
\hline PUFA (g/d) & $28 \cdot 8$ & $7 \cdot 0$ & $27 \cdot 6$ & 6.6 & 0.45 \\
\hline MUFA $(g / d)$ & 23.5 & 6.4 & 24.2 & $7 \cdot 2$ & 0.62 \\
\hline Cholesterol (mg/d) & $189 \cdot 3$ & 98.0 & 215.9 & $132 \cdot 7$ & 0.33 \\
\hline $\operatorname{TDF}(\mathrm{g} / \mathrm{d})$ & 19.0 & $4 \cdot 8$ & $17 \cdot 8$ & $5 \cdot 0$ & 0.28 \\
\hline $\mathrm{Fe}(\mathrm{mg} / \mathrm{d})$ & $15 \cdot 2$ & 3.3 & 15.5 & $2 \cdot 9$ & 0.66 \\
\hline $\mathrm{Mg}(\mathrm{mg} / \mathrm{d})$ & 292.4 & $79 \cdot 4$ & 283.6 & 78.1 & 0.63 \\
\hline $\mathrm{Zn}(\mathrm{mg} / \mathrm{d})$ & $10 \cdot 0$ & $3 \cdot 1$ & $10 \cdot 4$ & $3 \cdot 3$ & 0.59 \\
\hline $\mathrm{Mn}(\mathrm{mg} / \mathrm{d})$ & $2 \cdot 2$ & 0.7 & $2 \cdot 3$ & 0.7 & 0.51 \\
\hline
\end{tabular}

TDF, total dietary fibre.

* Obtained from independent $t$ test. 
After 6 weeks of intervention, compared with the placebo, synbiotic supplementation led to a significant decrease in serum insulin levels $(-1.5$ (SD 5.9) $v .+4 \cdot 8(\mathrm{sD} 11.5) \mu \mathrm{IU} / \mathrm{ml}, P=0.005)$, HOMA-IR $(-0 \cdot 4(\mathrm{SD} 1 \cdot 3) v .+1 \cdot 1(\mathrm{SD} 2 \cdot 7), P=0 \cdot 003)$ and HOMA-B $(-5 \cdot 1$ (sD 24.2) $v .+18.9$ (sD 45.6), $P=0.008)$ and a significant increase in QUICKI (+0.01 (SD 0.01) $v .-0.007$ (SD 0.02), $P=0.02$ ) (Table 3$)$. In addition, synbiotic intake significantly decreased serum TAG $(-14.8(\mathrm{sD} 56 \cdot 5) v .+30 \cdot 4(\mathrm{sD} 37 \cdot 8) \mathrm{mg} / \mathrm{dl}, P<0 \cdot 001)$ and VLDL-cholesterol concentrations ( -3.0 (sD 11.3) $v .+6 \cdot 1$ (sD 7.6) $\mathrm{mg} / \mathrm{dl}, P<0.001$ ) compared with the placebo. We did not observe any significant change after synbiotic supplementation on FPG and other lipid profiles.

Baseline concentrations of total cholesterol differed significantly between the two groups. Therefore, baseline concentrations of biochemical variables, maternal age and BMI at baseline were controlled for the analyses. However, after this adjustment, no significant changes in our findings were observed (Table 4).

\section{Discussion}

In the current study, we investigated the effects of synbiotic supplementation on markers of insulin metabolism and lipid profiles in patients with GDM. We found that the intake of probiotic supplements for 6 weeks among patients with GDM had beneficial effects on markers of insulin metabolism, TAG and VLDL-cholesterol concentrations; however, it did not influence FPG and other lipid profiles.

GDM women are susceptible to insulin resistance, dyslipidaemia and increased risk of adverse short- and long-term health outcomes ${ }^{(19)}$. This is therefore a good group to target with lifestyle interventions including synbiotic supplementation during pregnancy. Our study showed that synbiotic supplementation for 6 weeks in GDM women resulted in a significant reduction in serum insulin concentrations, HOMA-IR and HOMA-B and a significant increase in QUICKI compared with the placebo, but FPG remained unchanged. Although the

Table 3. Markers of insulin metabolism and lipid profiles at study baseline and after 6 weeks of intervention in patients with gestational diabetes mellitus who received either synbiotic supplements or placebo

(Mean values and standard deviations)

\begin{tabular}{|c|c|c|c|c|c|c|c|c|c|c|c|c|c|c|c|}
\hline & \multicolumn{7}{|c|}{ Placebo group $(n 35)$} & \multicolumn{7}{|c|}{ Synbiotic group ( $n$ 35) } & \multirow[b]{3}{*}{$P \dagger$} \\
\hline & \multicolumn{2}{|c|}{ Baseline } & \multicolumn{2}{|c|}{ End of trial } & \multicolumn{2}{|c|}{ Change } & \multirow[b]{2}{*}{$P^{\star}$} & \multicolumn{2}{|c|}{ Baseline } & \multicolumn{2}{|c|}{ End of trial } & \multicolumn{2}{|c|}{ Change } & \multirow[b]{2}{*}{$P^{\star}$} & \\
\hline & Mean & SD & Mean & SD & Mean & SD & & Mean & SD & Mean & SD & Mean & SD & & \\
\hline FPG (mg/dl) & $92 \cdot 1$ & $9 \cdot 2$ & 93.5 & $10 \cdot 3$ & 1.4 & 11.4 & 0.49 & $96 \cdot 2$ & $8 \cdot 0$ & 94.5 & 8.4 & $-1 \cdot 7$ & $9 \cdot 3$ & 0.27 & 0.22 \\
\hline Insulin $(\mu \mathrm{lU} / \mathrm{ml})$ & $13 \cdot 3$ & 5.4 & $18 \cdot 1$ & $12 \cdot 6$ & 4.8 & 11.5 & 0.01 & $13 \cdot 1$ & $7 \cdot 1$ & 11.6 & $3 \cdot 8$ & -1.5 & 5.9 & 0.13 & 0.005 \\
\hline HOMA-IR & $3 \cdot 1$ & 1.4 & 4.2 & $2 \cdot 8$ & $1 \cdot 1$ & $2 \cdot 7$ & 0.01 & 3.1 & $1 \cdot 7$ & $2 \cdot 7$ & 1.0 & -0.4 & 1.3 & 0.08 & 0.003 \\
\hline HOMA-B & $48 \cdot 1$ & $19 \cdot 4$ & $67 \cdot 0$ & 51.4 & 18.9 & $45 \cdot 6$ & 0.02 & $45 \cdot 8$ & $27 \cdot 0$ & $40 \cdot 7$ & $13 \cdot 8$ & $-5 \cdot 1$ & 24.2 & 0.12 & 0.008 \\
\hline QUICKI & 0.32 & 0.02 & 0.32 & 0.02 & -0.007 & 0.02 & 0.11 & 0.32 & 0.02 & 0.33 & 0.02 & 0.01 & 0.01 & $0 \cdot 18$ & 0.02 \\
\hline TAG (mg/dl) & $181 \cdot 1$ & 62.9 & 210.5 & $72 \cdot 1$ & $30 \cdot 4$ & 37.8 & $<0.001$ & $171 \cdot 3$ & 91.9 & $156 \cdot 5$ & 81.5 & $-14 \cdot 8$ & 56.5 & 0.13 & $<0.001$ \\
\hline VLDL-cholesterol (mg/dl) & $36 \cdot 0$ & $12 \cdot 6$ & $42 \cdot 1$ & 14.4 & $6 \cdot 1$ & $7 \cdot 6$ & $<0.001$ & 34.3 & 18.4 & 31.3 & $16 \cdot 3$ & -3.0 & $11 \cdot 3$ & 0.13 & $<0.001$ \\
\hline Total cholesterol (mg/dl) & $200 \cdot 0$ & $42 \cdot 4$ & $205 \cdot 2$ & 43.8 & $5 \cdot 2$ & $18 \cdot 7$ & 0.11 & 173.5 & $45 \cdot 3$ & 173.9 & $42 \cdot 1$ & 0.4 & 28.0 & 0.94 & 0.39 \\
\hline LDL-cholesterol (mg/dl) & $107 \cdot 0$ & $30 \cdot 1$ & $108 \cdot 2$ & $32 \cdot 0$ & $1 \cdot 2$ & $15 \cdot 1$ & 0.64 & $92 \cdot 2$ & $34 \cdot 2$ & 95.8 & $31 \cdot 3$ & 3.6 & $26 \cdot 1$ & 0.42 & 0.64 \\
\hline HDL-cholesterol (mg/dl) & 56.9 & $12 \cdot 0$ & 54.8 & $10 \cdot 8$ & $-2 \cdot 1$ & $7 \cdot 1$ & 0.08 & $47 \cdot 0$ & $9 \cdot 1$ & $46 \cdot 8$ & $8 \cdot 1$ & -0.2 & $6 \cdot 1$ & 0.80 & 0.22 \\
\hline
\end{tabular}

FPG, fasting plasma glucose; HOMA-IR, homoeostasis model assessment for estimated insulin resistance; HOMA-B, homoeostasis model assessment for estimated B cell function; QUICKI, quantitative insulin sensitivity check index.

* Obtained from paired-sample $t$ tests.

† Obtained from repeated-measures ANOVA test.

Table 4. Adjusted changes in metabolic variables in patients with gestational diabetes mellitus who received either synbiotic supplements or placebo

(Mean values with their standard errors)

\begin{tabular}{|c|c|c|c|c|c|}
\hline & \multicolumn{2}{|c|}{ Placebo group ( $n$ 35) } & \multicolumn{2}{|c|}{ Synbiotic group (n 35) } & \multirow[b]{2}{*}{$P^{\star}$} \\
\hline & Mean & SE & Mean & $\mathrm{SE}$ & \\
\hline FPG (mg/dl) & 0.02 & 1.6 & -0.4 & 1.5 & 0.83 \\
\hline Insulin $(\mu \mathrm{lU} / \mathrm{ml})$ & 4.9 & 1.5 & -1.6 & 1.5 & 0.003 \\
\hline HOMA-IR & $1 \cdot 1$ & 0.3 & -0.4 & 0.3 & 0.003 \\
\hline HOMA-B & $19 \cdot 3$ & $6 \cdot 1$ & $-5 \cdot 6$ & $6 \cdot 1$ & 0.005 \\
\hline QUICKI & -0.008 & 0.003 & 0.004 & 0.003 & 0.01 \\
\hline TAG (mg/dl) & 31.5 & $7 \cdot 6$ & $-15 \cdot 9$ & 7.6 & $<0.001$ \\
\hline VLDL-cholesterol $(\mathrm{mg} / \mathrm{dl})$ & $6 \cdot 3$ & 1.5 & $-3 \cdot 1$ & 1.5 & $<0.001$ \\
\hline Total cholesterol (mg/dl) & 7.5 & 3.9 & $-2 \cdot 0$ & 3.9 & 0.10 \\
\hline LDL-cholesterol (mg/dl) & 3.0 & 3.5 & 1.8 & 3.5 & 0.81 \\
\hline HDL-cholesterol (mg/dl) & -0.7 & 1.0 & -1.6 & 1.0 & 0.54 \\
\hline
\end{tabular}

FPG, fasting plasma glucose; HOMA-IR, homoeostasis model assessment for estimated insulin resistance; HOMA-B, homoeostasis model assessment

for estimated B cell function; QUICKI, quantitative insulin sensitivity check index.

* Obtained from ANCOVA test adjustment for baseline values plus maternal age and baseline BMI. 
beneficial effects of synbiotic intake on markers of insulin resistance on patients without GDM and animal models have been evaluated, to the best of our knowledge, this study is the first to assess the favourable effects of synbiotic supplementation on markers of insulin resistance in patients with GDM. In agreement with our findings, synbiotic supplementation containing 200 million of seven strains of friendly bacteria plus FOS for 28 weeks in subjects with the metabolic syndrome led to significant improvements in insulin resistance parameters ${ }^{(8)}$. In addition, Malaguarnera et al. ${ }^{(20)}$ noticed a significant reduction in the HOMA-IR following intake of Bifidobacterium longum plus FOS for 24 weeks among patients with non-alcoholic steatohepatitis, but no significant changes in serum insulin levels were observed. In another study, Yadav et al. ${ }^{(21)}$ observed that probiotic dahi-supplemented diet significantly delayed the onset of glucose intolerance, hyperglycaemia and hyperinsulinaemia in high fructose-fed rats. However, a few researchers were unable to observe such beneficial effects of probiotic supplementation on glycaemic status. For instance, a probiotic capsule intervention containing $10^{9} \mathrm{CFU}$ of L. salivarius among GDM women for 4-6 weeks had no impact on glycaemic control ${ }^{(22)}$. Hyperinsulinaemia and hyperglycaemia in GDM can result in progression to T2DM later in life and neonatal complications ${ }^{(23,24)}$. Synbiotic intake might improve glucose homoeostasis parameters through modification of gut flora, reduction of endotoxin levels, reduction of production and absorption of intestinal toxins, elevation of faecal $\mathrm{pH}^{(25)}$ and reduction of pro-inflammatory cytokine production ${ }^{(26)}$.

This study demonstrated that taking synbiotic supplements for 6 weeks by GDM women was associated with a significant reduction in serum TAG and VLDL-cholesterol concentrations, but did not influence other lipid profiles. We have previously shown that $\beta$-carotene fortified synbiotic food intake containing L. sporogenes $\left(27 \times 10^{7} \mathrm{CFU}\right)$ and $2.7 \mathrm{~g}$ inulin in patients with T2DM for 6 weeks decreased serum TAG and VLDL-cholesterol concentrations; however, it did not affect other lipid profiles ${ }^{(27)}$. Furthermore, synbiotic supplementation containing L. casei, Lactobacillus r.hamnosus, Streptococcus thermophilus, Bifidobacterium breve, L. acidophilus, Bifidobacterium longum and Lactobacillus bulgaricus $\left(2 \cdot 0 \times 10^{8} \mathrm{CFU}\right.$ each) plus FOS among obese children for 8 weeks resulted in a significant decrease in serum TAG and total- and LDL-cholesterol levels ${ }^{(28)}$. In another study, Schaafsma et al. ${ }^{(29)}$ observed that a combination of probiotic $L$. acidophilus plus FOS supplementation significantly reduced total-cholesterol, LDL-cholesterol and LDL-cholesterol/ HDL-cholesterol levels, while it did not influence TAG levels. In a similar study on men and women with hypercholesterolaemia, a significant reduction in total- and LDL-cholesterol concentrations was seen after 12 weeks of supplementation with a combination of $L$. acidophilus plus prebiotic inulin ${ }^{(30)}$. Both increased maternal TAG and free fatty acid levels in GDM women correlated with fetal growth during pregnancy and with neonatal anthropometric measures ${ }^{(31)}$. In addition, previous studies have reported that maternal lipid profile alterations are associated with complications such as macrossomia ${ }^{(32)}$, preeclampsia ${ }^{(33)}$ and preterm birth ${ }^{(34)}$. Different study designs, characteristics of study persons, different dosages, types and quality of the used probiotic bacteria and inulin as well as duration of the intervention are some reasons for discrepant findings. Synbiotic intake may decrease TAG and VLDL-cholesterol concentrations by lipolysis of TAG and transform TAG-rich particles into small particles ${ }^{(35)}$, suppressing the NF- $\kappa$ light-chain-enhancer of activated $\mathrm{B}$ cells pathway ${ }^{(36)}$ and gut microbiota-SCFA-hormone axis $^{(37)}$. In addition, probiotic and inulin intake may improve insulin resistance and lipid profiles through up-regulation of the PPAR- $\gamma$ gene ${ }^{(38,39)}$. In their study, Wang et $a l^{(40)}$ observed that $L$. case $i$ treatment significantly increased the expression of PPAR- $\gamma$ gene in a rat model of acute liver failure induced by lipopolysaccharide and D-galactosamine for $30 \mathrm{~d}$. PPAR- $\gamma$ is a member of the nuclear receptor superfamily and regulates the expressions of several genes encoding proteins involved in adipocyte differentiation, fatty acid storage and glucose metabolism ${ }^{(41)}$. Down-regulation in the expressions of hepatic genes involved in lipogenesis and fatty acid elongation/desaturation by inulin may also result in improvement in lipid profiles ${ }^{(42)}$.

Limitations of the current study include the absence of faecal sample data to demonstrate transit of the specific probiotic through the gastrointestinal tracts of study subjects in the intervention group. In addition, we did not use the faecal samples for colony count of specific probiotics. Because of funding limitations, we did not evaluate the expressed levels of related variables with insulin resistance and lipid profiles.

Overall, the results of this study demonstrate that taking synbiotic supplements for 6 weeks by patients with GDM had beneficial effects on markers of insulin metabolism, TAG and VLDL-cholesterol concentrations; however, it did not affect FPG and other lipid profiles. This suggests that synbiotic supplementation may confer advantageous therapeutic potential for patients with GDM. Further research is needed in other patients and for longer periods to determine the safety of this supplemental approach. In addition, further studies are needed to evaluate the expressed levels of related variables with insulin resistance and lipid profiles to explore the plausible mechanism and confirm our findings.

\section{Acknowledgements}

The present study was funded by a grant from the Vicechancellor for Research, AUMS, and Iran. The authors would like to thank the staff of Kosar Clinic (Arak, Iran) for their assistance on this project.

Z. A. contributed to the conception, design, statistical analysis and drafting of the manuscript. S. A., M. J., M. T.-E. and P. J. contributed to the conception, data collection and manuscript drafting. The final version was confirmed by all authors before submission.

The authors declare that there are no conflicts of interest.

\section{References}

1. Zhang C, Rawal S \& Chong YS (2016) Risk factors for gestational diabetes: is prevention possible? Diabetologia $\mathbf{5 9}$, $1385-1390$

2. Carpenter MW (2007) Gestational diabetes, pregnancy hypertension, and late vascular disease. Diabetes Care 30, Suppl. 2, S246-S250. 
3. Greenway F, Wang S \& Heiman M (2014) A novel cobiotic containing a prebiotic and an antioxidant augments the glucose control and gastrointestinal tolerability of metformin: a case report. Benef Microbes 5, 29-32.

4. Koren O, Goodrich JK, Cullender TC, et al. (2012) Host remodeling of the gut microbiome and metabolic changes during pregnancy. Cell 150, 470-480.

5. Fuller R (1991) Probiotics in human medicine. Gut 32, 439-442.

6. Gibson GR \& Roberfroid MB (1995) Dietary modulation of the human colonic microbiota: introducing the concept of prebiotics. J Nutr 125, 1401-1412.

7. Shakeri H, Hadaegh H, Abedi F, et al. (2014) Consumption of synbiotic bread decreases triacylglycerol and VLDL levels while increasing HDL levels in serum from patients with type-2 diabetes. Lipids $\mathbf{4 9}$, 695-701.

8. Eslamparast T, Zamani F, Hekmatdoost A, et al. (2014) Effects of synbiotic supplementation on insulin resistance in subjects with the metabolic syndrome: a randomised, double-blind, placebo-controlled pilot study. Br J Nutr 112, 438-445.

9. Taghizadeh M \& Asemi Z (2014) Effects of synbiotic food consumption on glycemic status and serum hs-CRP in pregnant women: a randomized controlled clinical trial. Hormones (Athens) 13, 398-406.

10. Rajkumar H, Kumar M, Das N, et al. (2015) Effect of probiotic Lactobacillus salivarius UBL S22 and prebiotic fructooligosaccharide on serum lipids, inflammatory markers, insulin sensitivity, and gut bacteria in healthy young volunteers: a randomized controlled single-blind pilot study. J Cardiovasc Pharmacol Ther 20, 289-298.

11. Vitali B, Ndagijimana M, Maccaferri S, et al. (2012) An in vitro evaluation of the effect of probiotics and prebiotics on the metabolic profile of human microbiota. Anaerobe 18, 386-391.

12. Voltolini C, Battersby S, Etherington SL, et al. (2012) A novel antiinflammatory role for the short-chain fatty acids in human labor. Endocrinology 153, 395-403.

13. American Diabetes Association (2014) Diagnosis and classification of diabetes mellitus. Diabetes Care 37, Suppl. 1, S81-S90.

14. Ainsworth BE, Haskell WL, Whitt MC, et al. (2000) Compendium of physical activities: an update of activity codes and MET intensities. Med Sci Sports Exerc 32, S498-S504.

15. Soccol CR, Vandenberghe LPdS, Spier MR, et al. (2010) The potential of probiotics: a review. Food Technol Biotech 48, 413-434.

16. Mohammadi AA, Jazayeri S, Khosravi-Darani K, et al. (2015) The effects of probiotics on mental health and hypothalamicpituitary-adrenal axis: a randomized, double-blind, placebocontrolled trial in petrochemical workers. Nutr Neurosci (Epublication ahead of print version 16 April 2015).

17. Benton D, Williams C \& Brown A (2007) Impact of consuming a milk drink containing a probiotic on mood and cognition. Eur J Clin Nutr 61, 355-361.

18. Pisprasert V, Ingram KH, Lopez-Davila MF, et al. (2013) Limitations in the use of indices using glucose and insulin levels to predict insulin sensitivity: impact of race and gender and superiority of the indices derived from oral glucose tolerance test in African Americans. Diabetes Care 36, 845-853.

19. Asemi Z, Samimi M, Tabassi Z, et al. (2014) The effect of DASH diet on pregnancy outcomes in gestational diabetes: a randomized controlled clinical trial. Eur J Clin Nutr $\mathbf{6 8}$, 490-495.

20. Malaguarnera M, Vacante M, Antic T, et al. (2012) Bifidobacterium longum with fructo-oligosaccharides in patients with non alcoholic steatohepatitis. Dig Dis Sci 57, 545-553.
21. Yadav H, Jain S \& Sinha PR (2007) Antidiabetic effect of probiotic dahi containing Lactobacillus acidophilus and Lactobacillus casei in high fructose fed rats. Nutrition 23, 62-68.

22. Lindsay KL, Brennan L, Kennelly MA, et al. (2015) Impact of probiotics in women with gestational diabetes mellitus on metabolic health: a randomized controlled trial. Am J Obstet Gynecol 212, 496.e1-496.e11.

23. Greenberg J (2011) The relationship between maternal glycemia and perinatal outcome. Obstet Gynecol 117, $1230-1231$.

24. Asemi Z, Karamali M \& Esmaillzadeh A (2014) Effects of calcium-vitamin D co-supplementation on glycaemic control, inflammation and oxidative stress in gestational diabetes: a randomised placebo-controlled trial. Diabetologia $\mathbf{5 7}$, 1798-1806.

25. Compare D, Coccoli P, Rocco A, et al. (2012) Gut-liver axis: the impact of gut microbiota on non alcoholic fatty liver disease. Nutr Metab Cardiovasc Dis 22, 471-476.

26. Li Z, Yang S, Lin H, et al. (2003) Probiotics and antibodies to TNF inhibit inflammatory activity and improve nonalcoholic fatty liver disease. Hepatology 37, 343-350.

27. Asemi Z, Alizadeh SA, Ahmad K, et al. (2016) Effects of betacarotene fortified synbiotic food on metabolic control of patients with type 2 diabetes mellitus: a double-blind randomized cross-over controlled clinical trial. Clin Nutr $\mathbf{3 5}$, 819-825.

28. Safavi M, Farajian S, Kelishadi R, et al. (2013) The effects of synbiotic supplementation on some cardio-metabolic risk factors in overweight and obese children: a randomized triple-masked controlled trial. Int J Food Sci Nutr 64, 687-693.

29. Schaafsma G, Meuling WJ, van Dokkum W, et al. (1998) Effects of a milk product, fermented by Lactobacillus acidophilus and with fructo-oligosaccharides added, on blood lipids in male volunteers. Eur J Clin Nutr 52, 436-440.

30. Ooi L-G, Ahmad R, Yuen K-H, et al. (2010) Lactobacillus acidophilus $\mathrm{CHO}-220$ and inulin reduced plasma total cholesterol and low-density lipoprotein cholesterol via alteration of lipid transporters. J Dairy Sci 93, 5048-5058.

31. Schaefer-Graf UM, Graf K, Kulbacka I, et al. (2008) Maternal lipids as strong determinants of fetal environment and growth in pregnancies with gestational diabetes mellitus. Diabetes Care 31, 1858-1863.

32. Kitajima M, Oka S, Yasuhi I, et al. (2001) Maternal serum triglyceride at 24-32 weeks' gestation and newborn weight in nondiabetic women with positive diabetic screens. Obstet Gynecol 97, 776-780.

33. Cekmen MB, Erbagci AB, Balat A, et al. (2003) Plasma lipid and lipoprotein concentrations in pregnancy induced hypertension. Clin Biochem 36, 575-578.

34. Vrijkotte TG, Krukziener N, Hutten BA, et al. (2012) Maternal lipid profile during early pregnancy and pregnancy complications and outcomes: the ABCD study. J Clin Endocrinol Metab 97, 3917-3925.

35. Matsuoka H, Miura A \& Hori K (2009) Symbiotic effects of a lipase-secreting bacterium, Burkholderia arboris SL1B1, and a glycerol-assimilating yeast, Candida cylindracea SL1B2, on triacylglycerol degradation. J Biosci Bioeng 107, 401-408.

36. Shi H, Kokoeva MV, Inouye K, et al. (2006) TLR4 links innate immunity and fatty acid-induced insulin resistance. $J$ Clin Invest 116, 3015-3025.

37. Yadav H, Lee JH, Lloyd J, et al. (2013) Beneficial metabolic effects of a probiotic via butyrate-induced GLP-1 hormone secretion. J Biol Chem 288, 25088-25097.

38. Deepak V, Ram Kumar Pandian S, Sivasubramaniam SD, et al. (2016) Optimization of anticancer exopolysaccharide 
production from probiotic Lactobacillus acidophilus by response surface methodology. Prep Biochem Biotechnol 46, 288-297.

39. Dewulf EM, Cani PD, Neyrinck AM, et al. (2011) Inulin-type fructans with prebiotic properties counteract GPR43 overexpression and PPARgamma-related adipogenesis in the white adipose tissue of high-fat diet-fed mice. J Nutr Biochem 22, 712-722.

40. Wang Y, Xie J, Li Y, et al. (2016) Probiotic Lactobacillus casei Zhang reduces pro-inflammatory cytokine production and hepatic inflammation in a rat model of acute liver failure. Eur J Nutr 55, 821-831.

41. Grindflek E, Sundvold H, Lien S, et al. (2000) Rapid communication: physical and genetic mapping of the peroxisome proliferator activated receptor gamma (PPAR-gamma) gene to porcine chromosome 13. J Anim Sci 78, 1391-1392.

42. Weitkunat K, Schumann S, Petzke KJ, et al. (2015) Effects of dietary inulin on bacterial growth, short-chain fatty acid production and hepatic lipid metabolism in gnotobiotic mice. J Nutr Biochem 26, 929-937. 